\title{
Human chromosome 15q11-q14 regions of rearrangements contain clusters of LCR15 duplicons
}

\author{
Miguel Angel Pujana ${ }^{1}$, Marga Nadal ${ }^{1}$, Miriam Guitart ${ }^{2}$, Lluís Armengol ${ }^{1}$, Mònica Gratacòs ${ }^{1}$ \\ and Xavier Estivill*,1
}

${ }^{1}$ Centre de Genètica Mèdica i Molecular - IRO, Hospital Duran i Reynals, Gran Vía km 2.7, 08907 L'Hospitalet de Llobregat, Barcelona, Spain; ${ }^{2}$ Corporació Parc Tauli, Laboratori de Genètica, Sabadell, Spain

Six breakpoint regions for rearrangements of human chromosome 15q11-q14 have been described. These rearrangements involve deletions found in approximately $70 \%$ of Prader-Willi or Angelman's syndrome patients (PWS, AS), duplications detected in some cases of autism, triplications and inverted duplications. HERC2-containing (HEct domain and RCC1 domain protein 2) segmental duplications or duplicons are present at two of these breakpoints (BP2 and BP3) mainly associated with deletions. We show here that clusters containing several copies of the human chromosome 15 low-copy repeat (LCR15) duplicon are located at each of the six described 15q11-q14 BPs. In addition, our results suggest the existence of breakpoints for large 15q11-q13 deletions in a proximal duplicon-containing clone. The study reveals that HERC2-containing duplicons (estimated on 50-400 kb) and LCR15 duplicons ( $15 \mathrm{~kb}$ on 15q11-q14) share the golgin-like protein (GLP) genomic sequence. Through the analysis of a human BAC library and public databases we have identified 36 LCR15 related sequences in the human genome, most (27) mapping to chromosome 15q and being transcribed. LCR15 analysis in non-human primates and age-sequence divergences support a recent origin of this family of segmental duplications through human speciation.

European Journal of Human Genetics (2002) 10, 26-35. DOI: 10.1038/sj/ejhg/5200760

Keywords: Prader-Willi syndrome; Angelman syndrome; inv dup(15); 15q11-q14; deletion/duplication; duplicons/segmental duplications

\section{Introduction}

Human chromosome $15 \mathrm{q} 11-\mathrm{q} 14$ is rearranged in several disorders and structural abnormalities. These include de novo deletions accounting for approximately $70 \%$ of cases of Prader-Willi (PWS; [MIM 176270]) and Angelman syndromes (AS; [MIM 105830]); ${ }^{1}$ 'inverted duplications', known as inv $\operatorname{dup}(15)$ or idic(15); ${ }^{2}$ and interstitial duplications, some present in cases of autism, ${ }^{3-5}$ or triplications. ${ }^{6-9}$ Some of the 15q11-q14 rearrangements are relatively frequent, with deletions on approximately $1 / 10000$ of live births and inv dup(15) in approximately 2/10 000 of live births. ${ }^{1,2}$ Amos-

*Correspondence: X Estivill, Centre de Genètica Mèdica i Molecular IRO, Hospital Duran i Reynals, Gran Vía km 2.7, 08907 L'Hospitalet de Llobregat, Barcelona, Spain. Tel: +34 932607406; Fax: +34 932607776; E-mail: estivill@iro.es

Received 17 August 2001; revised 12 October 2001; accepted 15 November 2001
Landgraf et al (1999) ${ }^{10}$ and Christian et al (1999) ${ }^{11}$ have shown that relatively large duplicon structures $(50-400 \mathrm{~kb})$ map to the breakpoint regions at $15 q 11$ and $15 q 13$. The hypothesis that $15 \mathrm{q}$ proximal rearrangements were due to repeated DNA sequences was first proposed by Donlon et al (1986), ${ }^{12}$ based on the identification of multiple copies of certain STSs. The 15q11-q13 duplicons identified by AmosLandgraf et al (1999) ${ }^{10}$ and Christian et al (1999) ${ }^{11}$ are associated to at least two of the previous described breakpoint regions, one proximal (BP2) and the other distal (BP3) (Figure 1). These duplicons contain at least seven different expressed sequences and are thought to have been generated approximately 20 million years ago. One of the duplicon-containing expressed sequences is the HERC2 gene (HEct domain and $R C c 1$ domain protein 2; also known as ERY-1), ${ }^{13}$ which has been found rearranged at the mRNA level in a PWS patient. ${ }^{10}$ Around 11 HERC2 copies have been identified in chromosomes 15 and 16 , but most of them are on 15q11-q13. ${ }^{14}$ 


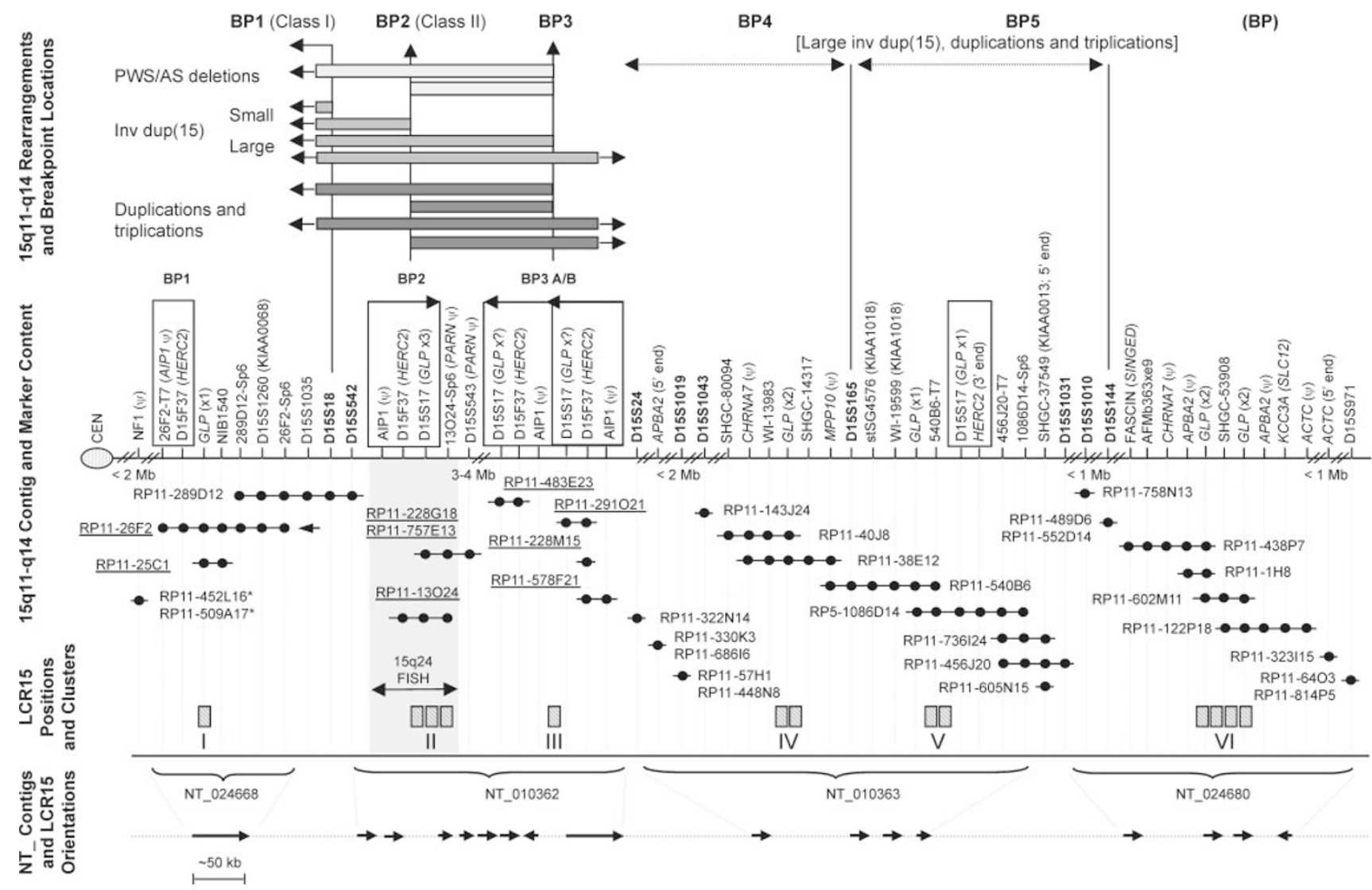

Figure 1 15q11-q14 chromosome rearrangements, breakpoint regions, and LCR15s clusters and copies. BP1 to BP5 breakpoints and class I (BP1) and class II (BP2) PWS/AS deletion types are shown. The (BP) located distally to marker D15S144, which has also been involved in rearrangements, ${ }^{22}$ has not been assigned a number. Contig and marker content of the 15q11-q14 region are shown. Reference markers previously reported in the definition of the breakpoint regions are shown in bold. The open rectangles indicate the $H E R C 2$-containing duplicon covered regions (not all of the markers included within these duplicons are shown). The D15S17 marker is included within the GLP genomic region. ( $\psi$ ) Symbol indicates pseudogenes. Abbreviations: RP11 (RPCI-11 BAC library) and CIT (California Institute of Technology BAC library). Underlined clones are those maps at HERC2-containing duplicons. According to sequence similarities <99\%, clones RP11-483E23 and RP11-291021/228M15/578F21 located at BP3 duplicons do not overlap, representing the two BP3 duplicons (A/B). An arrow indicates the position of the clone shown in Figure 4. Asterisks indicate clones located at a different position than expected from the NT_contigs. Filled circles indicate the presence of markers based on PCR and BLASTN analysis. The relative location of the different LCR15 groups (I to $\mathrm{VI}$ ) and copies is shown as vertical dashed boxes. The shaded region corresponds to FISH co-hybridisation on $15 q 24 .{ }^{11}$ The exact number of LCR15 copies located on BP3A/B is difficult to assign due to the complexity of the zone (x?). The exact number of these repeats will not be known until the complete sequence of these zones is achieved. The only locations where we have detected the D15S17 marker (a portion of the GLP genomic sequence) are BP2, BP3A/B and between RP11540B6 and RP5-1086D14 clones. For the rest of the LCR15 clusters the presence of other GLP sequences is indicated ( $x$ number of copies). Distances between duplicons on the NT_ contigs are not scaled. Putative duplicon orientations inferred from dot alignments are shown below.

BP2 refers to breakpoint site of class II PWS/AS patients where proximal microsatellite markers D15S18, D15S541 and D15S542 are not deleted. This is present in around half of the cases. In contrast, class I patients present a deletion of these markers and the respective breakpoint has been named BP1, being more proximal than $\mathrm{BP} 2$. These two breakpoint regions have been shown to be involved in similar frequencies in small inv dup(15) rearrangements and also in 15q11-q14 duplications and triplications. ${ }^{4,9,15-18}$ Three distal breakpoints have been described in 15q11-q14 rearrangements.
BP3, located at 15q13, is the most common distal breakpoint, accounting for the majority of cases of PWS/AS deletions and inv dup(15). The localisation of this breakpoint was established between markers D15S12 and D15S24. ${ }^{19-21}$ Two other breakpoint regions named BP4 and BP5 have been mapped distally to BP3, between markers D15S24 and D15S144, and seem to be implicated in cases of large 15q11-q14 duplications or triplications. ${ }^{4,5,9,18,20,21}$ These distal regions appear to be more complex since breakpoint variability has been described (BP located distal to 
D15S144). ${ }^{9,22}$ These observations would suggest the existence of different repeat sequences involved in rearrangements affecting these regions.

We describe here the identification of several copies of chromosome 15 low-copy repeats (LCR15s) or duplicons at the 15q11-q14 regions of rearrangements. We reveal that the LCR15s have sequence elements in common with the HERC2-containing duplicons. The specific distribution and clustering of the LCR15s on 15q11-q14 could provide a molecular basis to understanding the rearrangements affecting these regions. This hypothesis is supported by the observation that other copies of LCR15s are located flanking a relatively common 15q24-q26 genomic mutation identified by our group. ${ }^{23}$

The results presented here are based on the analysis of the public human sequence ${ }^{24}$ and on experimental data. The non-overlapping contigs for the 15q11-q14 breakpoint and LCR15 clusters shown in Figure 1 were built through the analysis of public raw data of each genomic clone sequence (not NT_contigs) and anchored using single copy markers derived from public or published 15q11-q14 maps. ${ }^{10,11,25-29}$ Subsequently, the contig was compared with the data present on NT_contigs. The experimental data obtained have allowed us to manage the unfinished status of the human sequence and the putative errors derived from the draft assembly of nearly identical sequence segmental duplications or duplicons.

\section{Materials and methods}

Cell cultures and DNA samples

PWS/AS cell lines (repository nos. GM11382, GM11385, GM11404 and GM11515) were purchased from the NIGMS Genetic Mutant Cell Repository. Seven additional PWS/AS cell cultures and DNA samples were obtained from patients attending at the Corporació Parc Taulí (CPT, Sabadell, Spain). Class I or class II PWS/AS deletion types were determined through D15S18 analysis. ${ }^{15}$ Class I deletions were revealed in one GM sample (GM11515) and four samples from the CPT. Macaca fasciluaris peripheral blood lymphocytes were kindly supplied by Dr Javier Guillén. Gorilla gorilla lymphoblasts were purchased from the ECACC General Cell Collection [EB (JC) cell line].

\section{Genomic clones and PCR analysis}

The RPC1-11 BAC library was purchased by the Resource Center within the German Human Genome Project (RZPD). Filters were screened by radioactive hybridisation (Megaprime DNA labelling system, Amersham, UK) using the REP471 probe. ${ }^{30}$ RPCI-11 clones identified through the analysis of public databases but not from the initial hybridisation of this library were also purchased from the RZPD center. Positive hybridisation clones were ordered, colony isolated, BAC-DNA extracted by the alkaline lysis method and subsequently PCR analysed. REP471 amplicon sets were designed from the LCR15 sequence on 15q26.1 (RP11-697E2; GenBank accession no. AC058820): REP471 amplicon; ${ }^{30}$ F1-R1 amplicon, F1 5'-gaagcaggctatagtatccaac-3' and R1 5'-ctacctgctgtatgccagaggttc-3', and Rc-R1 amplicon, using forward primer Rc 5'-gacttgcaatcatgaaaacca-3'. The F1R1 fragment (974 bp) includes the REP471 amplicon (135 bp) and the Rc-R1 amplicon (369 bp), which overlap at the Rc primer sequence (it is identical to the reverse primer sequence of the REP471 amplicon). All the clones studied that showed F1-R1 amplification also showed Rc-R1 amplification. Designed primer sets in order to obtain probes 1,3 and 5 were: probe $1 \mathrm{~F} 5^{\prime}$-tgtttagttcatggtcagacg-3' and $\mathrm{R} 5^{\prime}$ ttagatcacattactctctgg-3'; probe 3, F 5'-ttgctgactccagtcatg- $3^{\prime}$ and R 5'-ttgtaaccaatacaaggactg-3'; and probe 5, F 5'ttggaaacgccttgagaac- $3^{\prime}$ and R 5'-cgtgttcctgtacagttcc-3'. Hybridisation conditions were standard in Church's buffer followed by stringency washes to $0.25-0.1 \times$ SSC and $0.1 \%$ SDS at $60-65^{\circ} \mathrm{C}$ for $30 \mathrm{~min}$. Primer sets (purchased from Life Technologies) for 15q11-q14 single copy markers were those found at GDB database. SSCP analysis was performed on $12.5 \%$ polyacrylamide gels and silver staining (CleanGel DNA Analysis kit, Pharmacia). PCR agarose gel-purified fragments (QIAGEN) were sequenced according to the BigDye $^{(1 \times}$ Terminator RR Mix protocol and analysed on an ABI 377 automated sequencer (Applied Biosystems, Inc.).

Bioinformatic resources and sequence analysis BLASTN $^{31}$ and NIX (Williams GW Woollard PM and Hingamp P: 'NIX: A Nucleotide Identification system at the HGMP-RC', http://www.hgmp.mrc.ac.uk/NIX/) programs were used to analyse public databases. Large genomic sequences were aligned by PipMaker and MultiPipMaker programs $^{32}$ (MultiPipMaker kindly supported by Dr Webb Miller). Relatively short sequences were aligned with the CLUSTAL W program. ${ }^{33}$

\section{FISH analysis}

BAC DNA minipreparations were labelled with either biotin16dUTP or digoxigenin-11dUTP (Boehringer Mannheim) by standard nick-translation reaction and FISH protocol was performed as described elsewhere. ${ }^{34}$ Slides were studied under a fluorescence microscope (AH3, Olympus) equipped with the appropriate filter set. Images were analysed with the Cytovision system (Applied Imaging Ltd.).

\section{Results}

LCR15 duplicon identification and localisation

Hybridisation of the RPCI-11 (RP11 abbreviated) BAC library with probe REP471, contained in duplicon LCR15 on $15 q 26.1,{ }^{30}$ gave approximately 400 positive clones. From the $25 \times$ human genome coverage of this library, a first estimation of the copy number of the LCR15 duplicon was sixteen. Two hundred and eighty-one of these clones were requested and analysed by PCR for the presence of the 
REP471 sequence using two sets of amplicons (REP471 and F1-R1). A total of 255 clones (91\%) were positive for one or two of these amplicons $(+/+45 \% ;+/-8 \%$ and $-/+38 \%)$. PCR negative clones $(-/-9 \%)$ could correspond to errors in reading filters from the hybridisation screening or clones containing more divergent sequences. These negative clones were not further analysed.

To further classify the positive clones we performed SSCP analysis of the REP471 and F1-R1 amplicons. We subsequently sequenced the PCR products showing different SSCP patterns allowing a classification of the clones in at least 22 groups (Table 1). Since this classification was based on PCR and SSCP analysis of a part of the LCR15 sequence, the 22 groups probably represent an underestimation of the total LCR15 copy number in the human genome. Nineteen of these 22 groups were localised on chromosome 15. Interestingly four of the groups map on 15q11-q14, one on 15q21q22, eight on 15q24, three on 15q25 and two on 15q26. Some probes gave multiple signals by FISH and several clones gave additional signals on 15q24 probably due to the high LCR15 copy number in this region (unpublished results). The analysis of a panel of cell hybrid DNAs containing single human chromosomes ${ }^{35}$ revealed the existence of REP471 amplicons on chromosomes 2 (GenBank accession no. AJ306999), 7 (group 14) (GenBank accession no. AJ306998), 12 (GenBank accession no. AJ307000) and Y (group 7) (GenBank accession nos. AJ306997, AJ307001 and AJ307003).

LCR15 duplicon sequences were also searched by BLASTN analysis against public databases. As queries we used sequences from clones RP11-2M12 (GenBank accession no. AC013486) and RP11-697E2 (GenBank accession no. AC058820), which contain LCR15 elements that we have previously localised on $15 \mathrm{q} 24$ and $15 \mathrm{q} 26 .{ }^{30}$ Positive sequences (cut off at $>\mathrm{e}-100$, representing approximately 90 different clones) were subsequently BLASTN analysed using the NIX program through all the GenBank databases. The results of these analyses were in agreement with our previous classification and show that LCR15s are mainly distributed along chromosome 15, in five different regions: 15q11-q14, 15q21-q22, 15q24, 15q25 and 15q26. Each of these regions contains different LCR15 copies, as determined by the presence of single copy markers mapping at different locations. For example, on 15q11-q14 we have found the most proximal marker NIB1540 in the LCR15-containing clone RP11-26F2, marker D15S543, which is located distally to the BP2 duplicon, in the LCR15-containing clones RP11228G18 and RP11-757E13, or the most distal KCC3A gene in the LCR15-containing clone RP11-122P18 (Figure 1).

The estimated total number of LCR15 related sequences in the human genome is of at least 36 copies. Most LCR15s (27 copies) belong to chromosome 15 , of which at least 13 are from the 15q11-q14 region (Figure 1 and Table 1 ). At least nine LCR15 related sequences have been annotated to other chromosomes $(2,5,7,9,10,12,16,19$ and Y), but the number of clones LCR15 positive clones for these other locations is smaller (up to three clones each, not shown) compared to those on chromosome 15.

Through sequence alignments we estimated the LCR15 length extent in approximately $15 \mathrm{~kb}$ on 15q11-14 and approximately $60 \mathrm{~kb}$ (without HERC2 similarities) on 15q25q26 and chromosome Y (>95\% sequence identity between them, not shown). LCR15 duplicons contain several nonprocessed or pseudogene sequences. High similarities to DNM1, CHRNA7, SH3P18 and GLP genes were detected in different duplicons. The length of the genomic region that could be covered by expressed sequences is of about $15 \mathrm{~kb}$. The GLP BLASTN search against the EST database division revealed a large number of expressed sequences that when aligned show the existence of at least 15 different overlapping sequences. Similar results were obtained with other sequences within the LCR15s, as for example for DNM1, which is transcribed in some LCR15s in the opposite direction with respect to GLP (not shown). The GLP pseudogene is the most representative sequence of LCR15 duplicons, in agreement with the results of the screening of the RPCI-11 BAC library with probe REP471, containing GLP. The differences in the extent of LCR15 sequences are in agreement with the distinct FISH patterns detailed on Table 1 when LCR15-containing clones were used as probes.

\section{Duplicons mapping to 15q11-q14 sites of rearrangements} The identification of LCR15-containing RPCI-11 clones, BLASTN analysis of the respective public sequences, PCR and FISH analysis allow us to identify six LCR15 clusters on 15q11-q14 (Figure 1). The assigned positions were revealed by PCR using single copy markers not included within the 15q11-q14 segmental duplications or any other duplicated sequences. The LCR15 clusters were divided in the different paralogous copies through sequence analysis that showed specific sequence differences. FISH analysis of class I and class II deletion samples allowed us to confirm the existence of LCR15 clusters located distally and proximally to the respective deletions. The LCR15 15q11-q14 constructed contig was then compared with the public NT_contigs of the region. The results confirmed the locations assigned except for proximal clones RP11-452L16 and RP11-509A17. These clones are contained within the NT-010362 contig (mapped distally, at $\mathrm{BP} 2 / 3$ ). However, our analysis indicates that these clones could map more proximal. They contain neurofibromatosis type 1 pseudogene sequences, which are known to be located close to the $15 \mathrm{q}$ centromer, ${ }^{25}$ and also present sequence similarities with other human chromosome pericentromeric regions $(1 q, 2 p, 16 p, 17 p$ and $22 q 11)$, as has also been described for $15 \mathrm{q}$ pericentromer. ${ }^{36}$ The putative misassignment of these clones within the NT_010362 contig is probably due to the fact that clone RP11-509A17 contain HERC2 similarities (but with $<99 \%$ sequence identity to other BP2/3 clones). The proximal position of these clones in our contig could suggest that additional HERC2-containing 
Table 1 Survey of LCR15 elements in the human genome. LCR15s identified from the RPCI-11 library and classified according to REP471/F1-R1 amplicons, SSCP and sequence analysis.

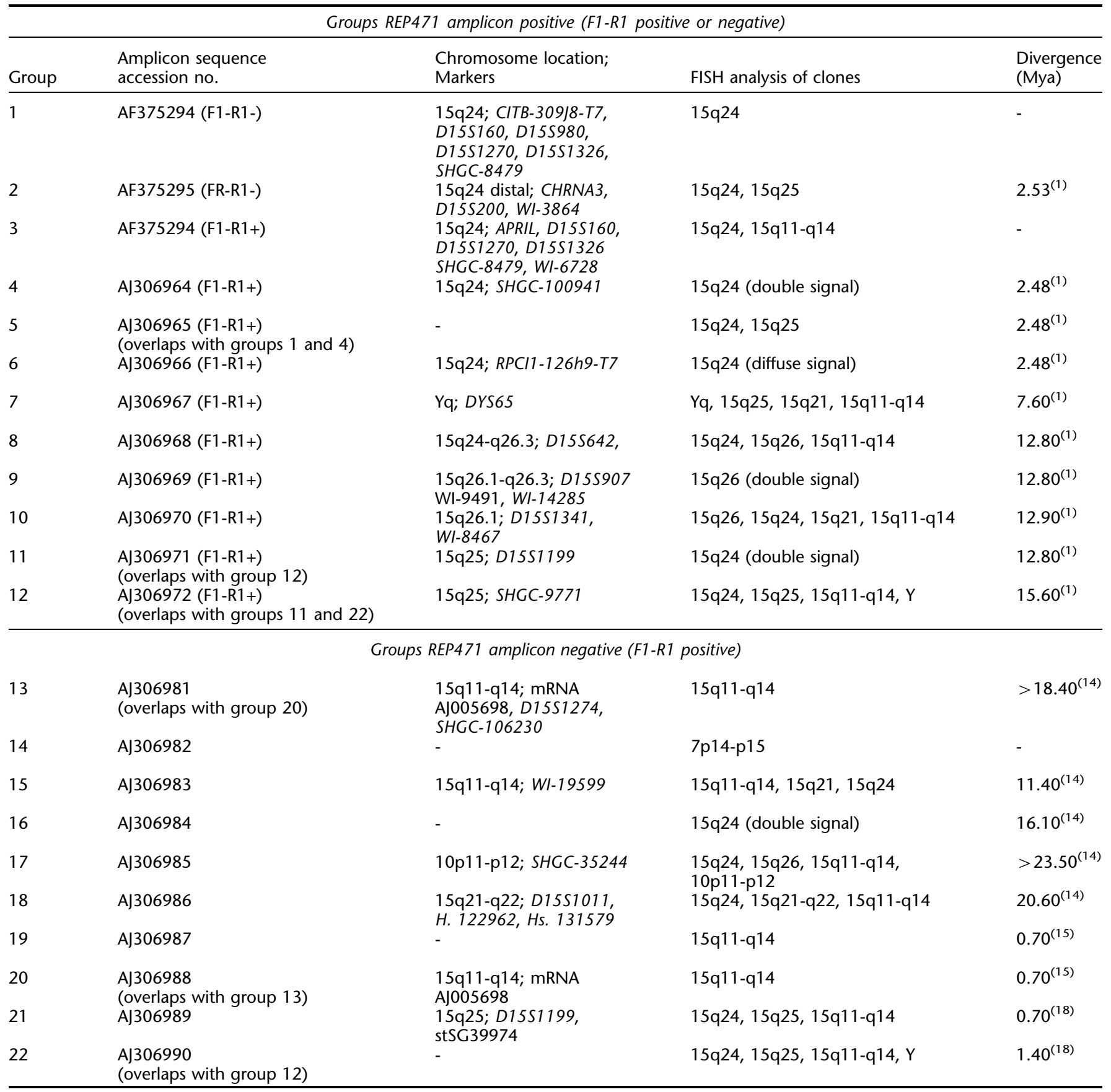

Single copy markers contained within identified clones were ascertained according to PCR and BLASTN analysis. The marker content defines the chromosomal location of each clone or group. The chromosome locations could not completely agree with FISH results, probally due to LCR15 cross-hybridisation and differences in repeat number between $15 q$ regions. FISH hybridisation results are ordered from more intense to less intense signals. Clone identification numbers belonging to each group are available from the authors. Sequence divergences were calculated with respect to: ${ }^{(1)}$ group $1 ;{ }^{(14)}$ group $14 ;{ }^{(15)}$ group $15 ;(18)$ group $18 .(>)$ Symbol indicates that divergences are greater due the presence of more than one LCR15 sequence or to the existence of deletions or insertions.

duplicons could map more centromeric than RP11-26F2 (Figure 1).
Multi-alignment analysis of raw data from the public sequence of genomic clones or NT_contigs shown that the 
A

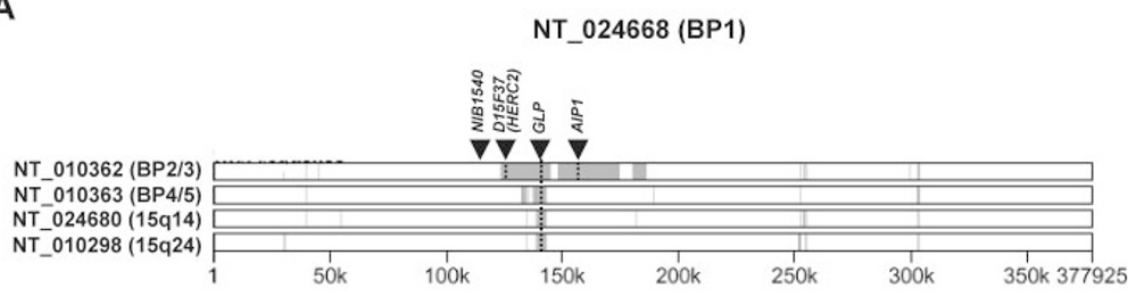

B

NT_024668 (BP1)
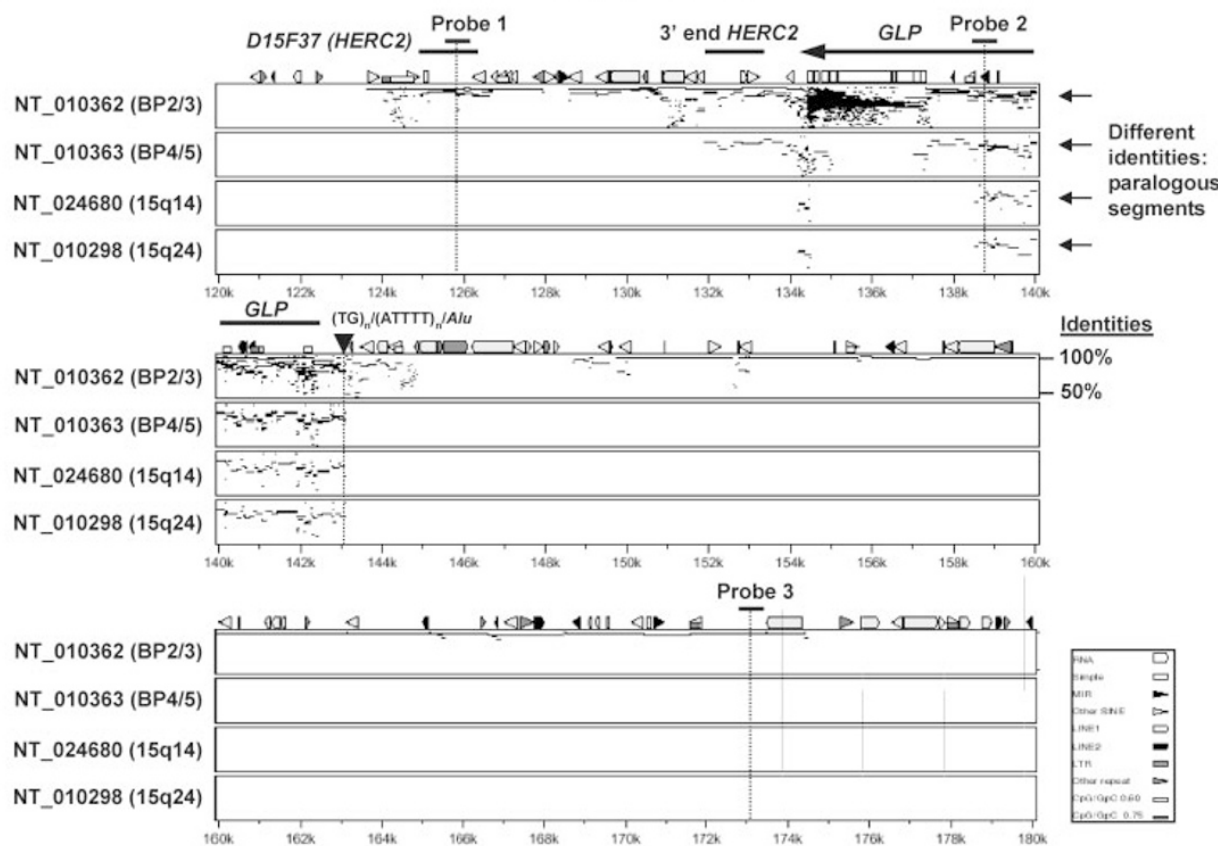

Figure 2 Multiple sequence alignment of the NT_024668 contig sequence (377,925 kb) on BP1 (15q11) against other LCR15containing contigs on 15q12-q14 and 15q24: overview of the NT_024668 sequence (A); detail of the duplicon-containing region (B). The locations and extensions of markers, HERC2 and GLP sequences are indicated. Since there is only one uninterrupted overlapping region, the NT_024668 contig only contains one duplicon element. The appearance of different alignments (different values of identity under the same regions) within the other contigs compared reveals the existence of more than one paralogous segment within them. Dashed lines indicate the location of the designed probes and the three different repeats revealed by the F1-R1 sequence analysis.

previously described HERC2 duplicons ${ }^{10,11}$ and LCR15 duplicons share the genomic region containing the GLP gene (Figure 2). Therefore, as the REP471 probe is included within this region, through the present analysis we have also identified the HERC2-containing duplicons at BP2 and BP3, but also at least one duplicon copy proximal to them, probably representing BP1. Furthermore, other LCR15 that contain GLP sequences but not HERC2 sequences were identified (Figures 1 and 2). This diversification of duplicons was also suggested through hybridisation analysis. High copy numbers for HERC2 and GLP sequences, but not for other duplicon specific regions, in the human genome were observed (Figure 3A). The pip and dot-plot analysis of NT_draft sequences also allowed us to suggest the duplicon orientations on 15q11-q14 (Figure 1). Nonetheless, these orientations should be taken with caution as they are based on the unfinished status of the public human genome sequence.

Through the BLASTN and FISH analysis of LCR15-containing 15q11-q14 clones we detected similarities with chromosome 16p11. These clones also contained HERC2 similarities. Copies of HERC2 have been described on chromosome 16 pericentromeric region. ${ }^{11,13,14}$ Sequence alignments show that the sequence responsible for this $16 \mathrm{p} 11$ similarity has a length of $13 \mathrm{~kb}$. This HERC2-containing 16p11 duplicon is at $35 \mathrm{~kb}$ from another unrelated segmental duplication included within the same public clone sequence (GenBank accession no. AC002041). ${ }^{36}$ 
Probe 1 (HERC2)

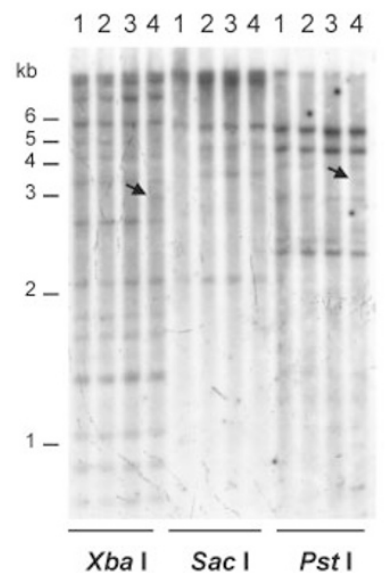

Probe 2 (GLP)
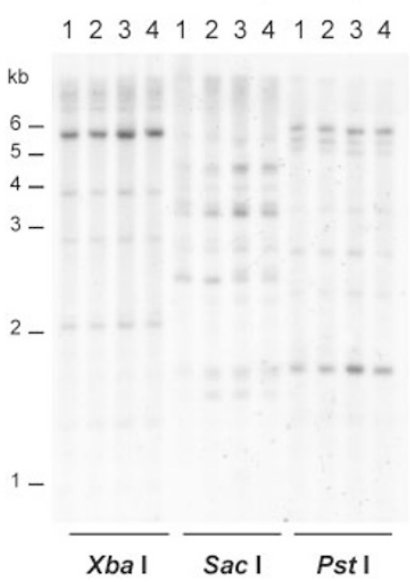

\section{Probe 3}

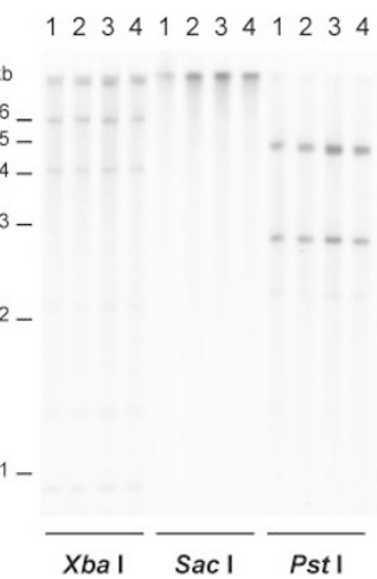

\section{REP471}

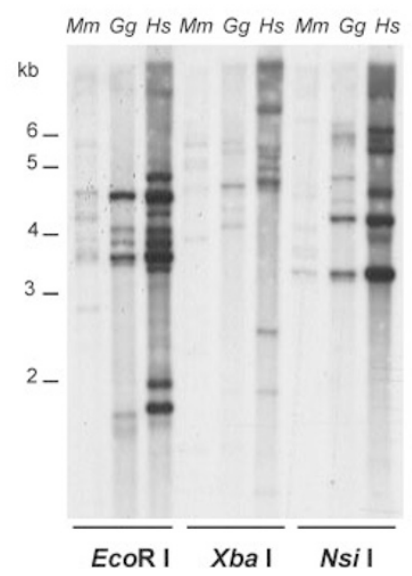

Figure 3 (A) Southern hybridisation results for three probes designed for the multiple alignment (Figure 2). Lane 1: GM11515; lane 2: GM11385; lane 3: control sample from the general population; and lane 4: GM11404. Arrows indicate the additional fragments in a high hybridisation background. (B) REP471 Southern hybridisation analysis of DNA from human (Hs) and non-human primates samples (Gg, Gorilla gorilla; $\mathrm{Mm}$, Macaca fascicularis).

The presence of several LCR15s at the end of the BP2HERC2 duplicon explains previous FISH reports giving signals on both $15 \mathrm{q} 11-\mathrm{q} 13$ and $15 \mathrm{q} 24 .{ }^{11}$ Two of these copies correspond to LCR15 groups 13 and 20, contained in clone RP11-13024 (GenBank accession no. AC016033) (Table 1). Since clone 147B6 (group 19) is positive for markers D15S15, D15S17 and 363H3L (not shown), the third LCR15 copy probably corresponds to this group.

Clone RP5-1086D14 (GenBank accession no. AC004460), within cluster IV, was positive for the 3 ' end of the HERC2 gene as described by Ji et al $^{14}$ but not for other HERC2-containing duplicon markers. This distal region to BP3 is highly rich in partially duplicated genes (CHRNA7, MPP10, APBA2 and $A C T C)$. Interestingly, sequence similarities with the CHRNA7 gene are also present in other LCR15 on chromosome 15q (not shown). These similarities include the previously reported duplicated sequences CHRNA7-DR1 and DR2 (GenBank accession nos. AF029838 and AF029839), ${ }^{29}$ suggesting that the LCR15 duplicon could have participated in the CHRNA7 partial duplication.

\section{Duplicon analysis in PWS/AS 15q deletion patients}

The existence of two different classes of 15q11-q13 deletions allowed us, by FISH analysis, to confirm the presence of LCR15 clusters on both sides of these rearrangements. For this purpose, we hybridised class I and II deleted chromosomes with different LCR15-containing clones: RP11-26F2 on BP1; RP11-13O24 and RP11-757E13 on BP2; RP11-291O21 and RP11-483E23 on BP3A/B; RP11-38E12 and RP11-40J8 on cluster IV; RP11-540B6 and RP5-1086D14 on cluster V; and RP11-122P18 and RP11-438P7 on cluster VI.

FISH analysis of class I samples (GM11515 and four additional samples obtained from the CPT) revealed a weaker signal on the deleted chromosome using probe RP11-26F2 (Figure 4). This observation suggests that the proximal breakpoint of this rearrangement could be located within the sequence of this clone, proximal to marker D15S18.

Analysis with probe 1 (HERC2 genomic region) revealed an additional fragment in two different DNA restriction digestions from the AS GM11404 cell line (Figure 3A). These extra fragments should correspond to a junction fragment due to the deletion affecting BP2 or BP3 regions in this patient or could represent a sequence polymorphism at one of the duplicated sequences detected by the probe. Amos-Landgraf et $a l^{10}$ have previously shown a possible HERC2 rearranged transcript in a PWS patient.

\section{LCR15 analysis in non-human primates}

Southern hybridisation results using the REP471 probe demonstrate the relatively recent evolutionary origin of the LCR15 sequences. This analysis was carried out with DNA from an Old World monkey (Macaca fascicularis) and one great ape species (Gorilla gorilla). A large number of LCR15 copies, albeit lower than in humans, were detected (Figure 3B). Interestingly, some of the fragments are shared by the three species compared here and in order to divergence from humans (approximately 20 Mya from M. fascicularis and approximately 7 Mya from G. gorilla). 
A

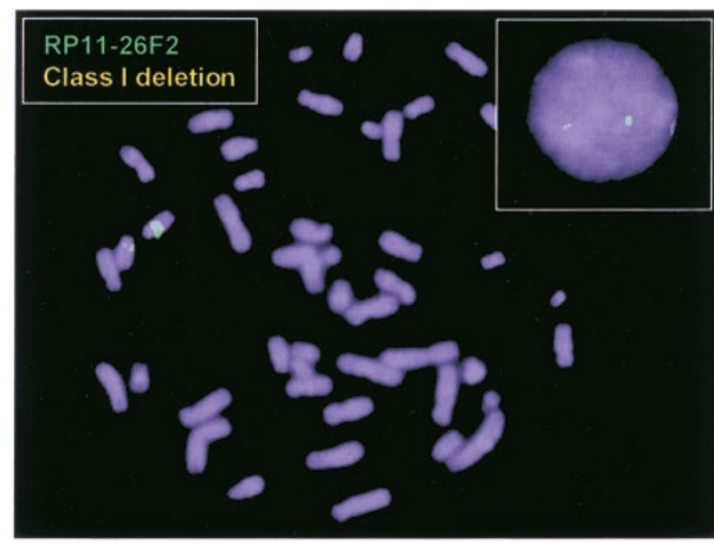

B

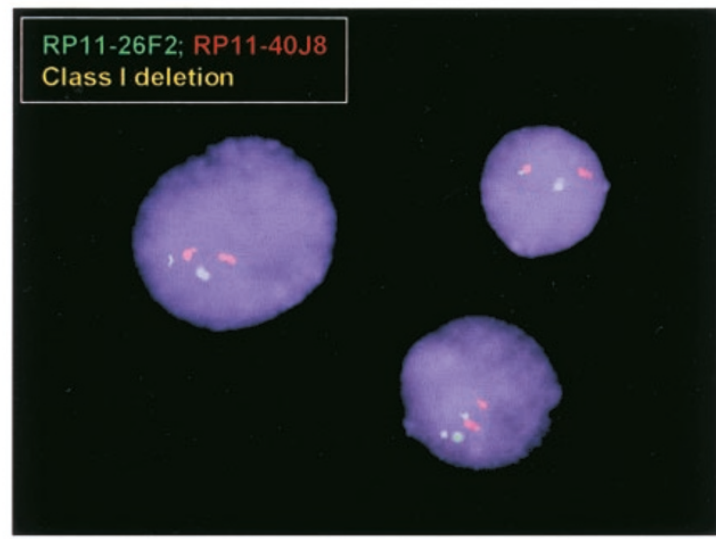

C

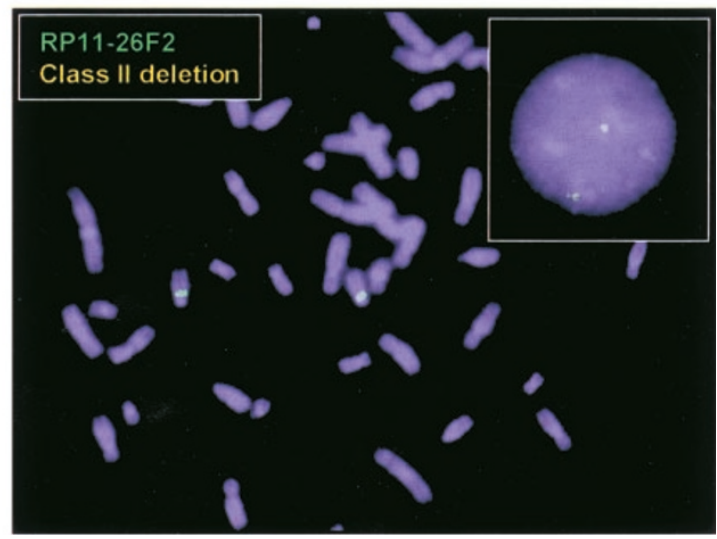

Figure 4 FISH hybridisation results of probe RP11-26F2 (BP1; Figure 1) on metaphase and interphase chromosomes from a class I deletion patient with AS (A); interphase chromosomes from the same patient hybridised with RP11-26F2 (green) and RP11-40J8 control probe (red; mapped on 15q13, see Figure 1 ; the metaphase chromosomes are not shown due to the proximity of the probes) (B); and class II deletion patient (C).

Database search for orthologous mouse GLP sequences only identified one mouse genomic clone (RP23-170C15; GenBank accession no. AC068494). This clone contains the dynamin 1 (Dnm1) mouse gene on chromosome 2p. This region is syntenic with the human chromosome 9q33-q34, where we have also identified LCR15 similarities. Since there is only one mouse Dnm1 sequence, it could be postulated that the first human LCR15 related sequence originated from 9q33-q34, where interestingly human DNM1 and GOLGA2 (golgi autoantigen A2) genes are closely located.

\section{Discussion}

Low copy repeat sequences containing non-processed genes or pseudogenes are known as 'duplicons'. ${ }^{37}$ Duplicons flank chromosome regions that undergo different types of human chromosomal rearrangements, such as deletions, duplications and inversions, which could occur through unequal homologous recombination events. These rearrangements seem to be promoted by the high sequence similarity between the duplicon copies and the common presence of non-processed or pseudogene expressed sequences within them. ${ }^{38,39}$

We reveal here the identification and localisation of duplicon elements in the six breakpoint regions described where 15q11q14 rearrangements occur. While duplicons characterised by the presence of HERC2 pseudogenes have been described for two of the defined breakpoints (BP2 and BP3) ${ }^{10,11}$ duplicon sequences have not been detected for the rest of 15q11-q14 BPs. Although the breakpoint regions described at the distal LCR15s positions (clusters IV, V and VI) are $>1 \mathrm{Mb}$ in size and although the LCR15 extent in these regions is of approximately $15 \mathrm{~kb}$, the presence of clusters (now extending the repeats to approximately 30-60 kb) at these particular locations could suggest a role in the rearrangements affecting these regions. Although a direct correlation seems to exist between the chromosome rearrangement size and the mediating duplicon extent, ${ }^{38,39}$ it is possible that other duplicon features could have an important role in promoting rearrangements. Nonetheless, the frequency of 15q11-q13 deletions, possibly involving large HERC2-containing duplicons, is higher than the described frequency for the large duplications where the LCR15 duplicons could be involved. The duplicon complexity of the 15q11-q14 region and the different rearrangement types that could be originated from them have a precedent on human chromosome 22q11 region. ${ }^{40,41}$ In addition to the putative role of LCR15 duplicons on mediating 15q11-q14 rearrangements, our group has identified other copies of LCR15s flanking a 15q24-q26 genomic mutation. $^{23}$

According to what has been stated above, we suggest here the delimitation of a class I proximal breakpoint in a newly identified duplicon-containing clone. However, as this result is only based on FISH hybridisation analysis of a few class I samples, a detailed FISH and molecular analysis of a larger number of these patients is required.

Other considerations could add more complexity to the 15q11-q14 region and rearrangements. Misalignment and crossing-over between different duplicons located on the three distal LCR15 clusters could lead to not yet detected submicroscopic duplications or deletions associated to disease traits linked to these regions, as for example a 
neurophysiological deficit present in schizophrenia ${ }^{42}$ or autism. $^{26}$ In addition, since LCR15s appear in clusters, it could be possible that a variable number of copies and/or orientations exist at specific positions in some chromosomes. These putative repeat differences could be associated to a lesser or higher susceptibility to 15q11-q14 chromosome rearrangements, in such a way as has been described for three different recurrent chromosome $8 \mathrm{p}$ rearrangements. ${ }^{43}$

Surprisingly, HERC2-containing duplicons and LCR15 duplicons are partially related since they share GLP gene sequences, which are characteristic of all LCR15s. Aside from chromosome 15q, we describe here that LCR15 copies also lie on chromosome Y. Detailed analysis of these copies has located them on Yq11.22, flanking the DYS7 marker (not shown). Interestingly, this marker has been reported to be recurrently deleted and duplicated. ${ }^{44}$ Recently, these golfinrelated duplicons have been described associated to the AZFc region. ${ }^{45}$

The LCR15 duplicon has a large number of copies, as compared to other duplicons described so far. Interestingly most of the LCR15 copies are located on chromosome 15. Our observation of high clustering of LCR15 in discrete and large regions of the chromosome suggests that LCR15 duplicon could be considered as a 'mobile' element in the evolution of this chromosome. Although other types of repeated sequences such as LINE elements show high mobility rates, ${ }^{46}$ the mobility capacity of duplicons is unknown. The common presence of pseudogene sequences within LCR15 copies suggests a role for an open DNA/ chromatin structure. This is a common feature for other described human duplicons and it has been suggested to facilitate recombination events. ${ }^{37-39}$

From the LCR15-containing clone classification described here, we have estimated the smallest sequence divergence to be as recent as 0.7 million years. On the other hand, the maximum values of estimated divergences are included within the primate evolution period (<63 Mya) ${ }^{47}$ Although these estimations are probably biased due to the specificity of the used PCR primers, they are also common for other described duplicon elements. ${ }^{14,37,39,48}$

In summary, we have shown here that the 15q11-q14 region contains a clustering of duplicons with a common signature. The present data clearly indicate the need to analyse not only the BP4 and BP5 in large 15q11-q14 rearrangements affecting these regions, but also the putative involvement of the proximal BP1 duplicon in class I PWS/AS patients. The complexity of chromosome regions containing duplicon elements may be difficult to assemble in sequenced regions of the genome. The draft sequences of the human genome have reported a large number of genomic regions that are duplicated within and between chromosomes. ${ }^{24,49}$ The anchored approach in sequencing the human genome will prove extremely valuable in resolving ambiguities ( $<99 \%$ sequence identity) due to the presence of duplicon sequences. Ultimately, the comparative analysis of the human genome with other duplicon-free genomes, as for example the mouse genome, should help with the correct assembly of the segmental duplications.

\section{EMBL/GenBank accession numbers}

AF375294, AF375295, AJ306964-AJ306972, AJ306981AJ307003.

\section{Acknowledgments}

We thank Dr Javier Guillén for providing peripheral blood lymphocytes from Macaca fascicularis. We thank Helena Kruyer for assistance in preparing the manuscript. This work was supported by La Marato de TV3 (98/1810), the European Union (BMH4-CT97-2284) and the Spanish Government (CICYT; SAF99-0092-CO2-01). MA Pujana is a fellow of the Centre de Regulació Genomica. L Armengol is a fellow of the Comissió Interdepartamental de Recerca i Innovació Tecnologica of the Generalitat de Catalunya.

\section{References}

1 Khan NL, Wood NW: Prader-Willi and Angelman syndromes: update on genetic mechanisms and diagnostic complexities. Curr Opin Neurol 1999; 12: 149-154.

2 Webb T, Clarke D, Hardy CA, Kilpatrick MW, Corbett J, Dahlitz M: A clinical, cytogenetic, and molecular study of 40 adults with the Prader-Willi syndrome. J Med Genet 1995; 32: 181-185.

3 Bundey S, Hardy C, Vickers S, Kilpatrick MW, Corbett JA: Duplicon of the 15q11-13 region in a patient with autism, epilepsy and ataxia. Dev Med Child Neurol 1994; 36: 736-742.

4 Browne CE, Dennis NR, Maher E et al: Inherited interstitial duplications of proximal 15q: genotype-phenotype correlations. Am J Hum Genet 1997; 61: $1342-1352$.

5 Cook Jr EH, Lindgren V, Leventhal BL et al: Autism or atypical autism in maternally but not paternally derived proximal $15 \mathrm{q}$ duplication. Am J Hum Genet 1997; 60: 928-934.

6 Schinzel AA, Brecevic L, Bernasconi F et al: Intrachromosomal triplication of 15q11-q13. J Med Genet 1994; 31: 798-803.

7 Cassidy S, Conroy J, Becker L, Schwartz S: Paternal triplication of 15q11-13 in a hypotonic, developmentally delayed child without Prader-Willi or Angelman syndrome. Am J Med Genet 1996; 62: $205-212$.

8 Long FL, Duckett DP, Billam LJ, Williams DK, Crolla JA: Triplication of 15q11-q13 with inv $\operatorname{dup}(15)$ in a female with developmental delay. J Med Genet 1998; 35: 425-428.

9 Ungaro P, Christian SL, Fantes JA et al: Molecular characterisation of four cases of intrachromosomal triplication of chromosome 15q11-q14. J Med Genet 2001; 38: 26-34.

10 Amos-Landgraf JM, Ji Y, Gottlieb W et al: Chromosome breakage in the Prader-Willi and Angelman syndromes involves recombination between large, transcribed repeats at proximal and distal breakpoints. Am J Hum Genet 1999; 65: 370-386.

11 Christian SL, Fantes JA, Mewborn SK, Huang B, Ledbetter DH: Large genomic duplicons map to sites of instability in the Prader-Willi/Angelman syndrome chromosome region (15q11q13). Hum Mol Genet 1999; 8: 1025 - 1037.

12 Donlon TA, Lalande M, Wyman A, Bruns G, Latt SA: Isolation of molecular probes associated with the chromosome 15 instability in the Prader-Willi syndrome. Proc Natl Acad Sci USA 1986; 83: $4408-4412$

$13 \mathrm{Ji} \mathrm{Y}$, Walkowicz MJ, Buiting $\mathrm{K}$ et al: The ancestral gene for transcribed, low-copy repeats in the Prader-Willi/Angelman region encodes a large protein implicated in protein trafficking, which is deficient in mice with neuromuscular and spermiogenic abnormalities. Hum Mol Genet 1999; 8: 533-542. 
14 Ji Y, Rebert NA, Joslin JM, Higgins MJ, Schultz RA, Nicholls RD: Structure of the highly conserved HERC2 gene and of multiple partially duplicated paralogs in human. Genome Res 2000; 10: $319-329$.

15 Knoll JH, Nicholls RD, Magenis RE et al: Angelman syndrome: three molecular classes identified with chromosome 15q11q13specific DNA markers. Am J Hum Genet 1990; 47: 149-155.

16 Christian SL, Robinson WP, Huang B et al: Molecular characterization of two proximal deletion breakpoint regions in both Prader-Willi and Angelman syndrome patients. Am J Hum Genet 1995; 57: 40-48.

17 Huang B, Crolla JA, Christian SL et al: Refined molecular characterization of the breakpoints in small inv dup(15) chromosomes. Hum Genet 1997; 99: 11-17.

18 Robinson WP, Horsthemke B, Leonard S et al: Report of the Third International Workshop on Human Chromosome 15 Mapping 1996. October 25-27, 1996 in Vancouver B.C., Canada. Cytogenet Cell Genet 1997; 76: 1- 13.

19 Cheng SD, Spinner NB, Zackai EH, Knoll JH: Cytogenetic and molecular characterization of inverted duplicated chromosomes 15 from 11 patients. Am J Hum Genet 1994; 55: 753 - 759.

20 Crolla JA, Harvey JF, Sitch FL, Dennis NR: Supernumerary marker 15 chromosomes: a clinical, molecular and FISH approach to diagnosis and prognosis. Hum Genet 1995; 95: $161-170$

21 Wandstrat AE, Leana-Cox J, Jenkins L, Schwartz S: Molecular cytogenetic evidence for a common breakpoint in the largest inverted duplications of chromosome 15. Am J Hum Genet 1998; 62: $925-936$.

22 Robinson WP, Dutly F, Nicholls RD et al: The mechanisms involved in formation of deletions and duplications of $15 \mathrm{q} 11$ q13. J Med Genet 1998; 35: 130-136.

23 Gratacòs M, Nadal M, Martín-Santos R et al: A polymorphic genomic duplication on human chromosome 15 is a susceptibility factor for panic and phobic disorders. Cell 2001; 106: $367-379$

24 Lander ES, Linton LM, Birren B et al: Initial sequencing and analysis of the human genome. Nature 2001; 409: 860-921.

25 Ritchie RJ, Mattei MG, Lalande M: A large polymorphic repeat in the pericentromeric region of human chromosome 15q11-q13 contains three partial gene duplications. Hum Mol Genet 1998; 7: $1253-1260$.

26 Maddox LO, Menold MM, Bass MP et al: Autistic disorder and chromosome 15q11-q13: construction and analysis of a BAC/ PAC contig. Genomics 1999; 62: 325-331.

27 Christian SL, Bhatt NK, Martin SA et al: Integrated YAC contig map of the Prader-Willi/Angelman region on chromosome $15 \mathrm{q} 11-\mathrm{q} 13$ with average STS spacing of $35 \mathrm{~kb}$. Genome Res 1998; 8: $146-157$

28 Morton CC, Christian SL, Donlon TA et al: Report of the fourth international workshop on human chromosome 15 mapping 1997. Cytogenet Cell Genet 1999; 84: 12 - 21.

29 Gault J, Robinson M, Berger R et al: Genomic organization and partial duplication of the human alpha7 neuronal nicotinic acetylcholine receptor gene (CHRNA7). Genomics 1998; 52: $173-185$.

30 Pujana MA, Nadal M, Gratacòs M et al: Additional complexity on human chromosome 15q: identification of a set of newly recognized duplicons (LCR15) on 15q11-q13, 15q24, and 15q26. Genome Res 2001; 11: 98-111.

31 Altschul SF, Gish W, Miller W, Myers EW, Lipman DJ: Basic local alignment search tool. J Mol Biol 1990; 215: 403-410.
32 Schwartz S, Zhang Z, Frazer KA et al: PipMaker - a web server for aligning two genomic DNA sequences. Genome Res 2000; 10: $577-586$.

33 Thompson JD, Higgins DG, Gibson TJ: CLUSTAL W: improving the sensitivity of progressive multiple sequence alignment through sequence weighting, position-specific gap penalties and weight matrix choice. Nucleic Acids Res 1994; 22: 4673 4680.

34 Nadal M, Moreno S, Pritchard M, Preciado MA, Estivill X, Ramos-Arroyo MA: Down syndrome: characterisation of a case with partial trisomy of chromosome 21 owing to a paternal balanced translocation $(15 ; 21)(\mathrm{q} 26 ; \mathrm{q} 22.1)$ by FISH. J Med Genet 1997; 34: 50-54.

35 Dubois BL, Naylor SL: Characterization of NIGMS human/ rodent somatic cell hybrid mapping panel 2 by PCR. Genomics 1993; 16: $315-319$.

36 Horvath JE, Schwartz S, Eichler EE: The mosaic structure of human pericentromeric DNA: a strategy for characterizing complex regions of the human genome. Genome Res 2000; 10: $839-852$.

37 Eichler EE: Masquerading repeats: paralogous pitfalls of the human genome. Genome Res 1998; 8: 758-762.

38 Lupski JR: Genomic disorders: structural features of the genome can lead to DNA rearrangements and human disease traits. Trends Genet 1998; 14: 417-422.

39 Ji Y, Eichler EE, Schwartz S, Nicholls RD: Structure of chromosomal duplicons and their role in mediating human genomic disorders. Genome Res 2000; 10: 597-610.

40 Shaikh TH, Kurahashi H, Saitta SC et al: Chromosome 22specific low copy repeats and the 22q11.2 deletion syndrome: genomic organization and deletion endpoint analysis. Hum $\mathrm{Mol}$ Genet 2000; 9: 489-501.

41 Edelmann L, Pandita RK, Spiteri E et al: A common molecular basis for rearrangement disorders on chromosome 22q11. Hum Mol Genet 1999; 8: 1157-1167.

42 Freedman R, Coon H, Myles-Worsley $\mathrm{M}$ et al: Linkage of a neurophysiological deficit in schizophrenia to a chromosome 15 locus. Proc Natl Acad Sci USA 1997; 94: 587-592.

43 Giglio S, Broman KW, Matsumoto N et al: Olfactory receptorgene clusters, genomic-inversion polymorphisms, and common chromosome rearrangements. Am J Hum Genet 2001; 68: 874883.

44 Jobling MA, Samara V, Pandya A et al: Recurrent duplication and deletion polymorphisms on the long arm of the Y chromosome in normal males. Hum Mol Genet 1996; 5: 1767-1775.

45 Kuroda-Kawaguchi T, Skaletsky H, Brown LG et al: The AZFc region of the $\mathrm{Y}$ chromosome features massive palindromes and uniform recurrent deletions in infertile men. Nature Genet 2001; 29: $279-286$.

46 Pickeral OK, Makalowski W, Boguski MS, Boeke JD: Frequent human genomic DNA transduction driven by LINE-1 retrotransposition. Genome Res 2001; 10: 411-415.

47 Goodman M: The genomic record of Humankind's evolutionary roots. Am J Hum Genet 1999; 64: 31 - 39.

48 Keller MP, Seifried BA, Chance PF: Molecular evolution of the CMT1A-REP region: a human- and chimpanzee-specific repeat. Mol Biol Evol 1999; 16: 1019-1026.

49 Venter JC, Adams MD, Myers EW et al: The sequence of the human genome. Science 2001; 291: 1304-1351. 